\section{Species and speciation}

SIR - In his valuable review of genetic differences that make us attribute individuals to different species, Coyne ${ }^{1}$ repeated claims about sympatric speciation and the biological species concept (BSC) which, I believe, are incorrect, although widely accepted.

'Neodarwinism' is not limited to the "view that species usually arise as the byproducts of evolution in geographically isolated populations" (allopatric speciation). Sympatric speciation within one population is equally "neodarwinian': both are caused by natural selection of genetic variation, although conditions of sympatric speciation are stringent (for example, only a few loci can be involved ${ }^{2}$ ), while isolated populations always diverge (but sometimes very slowly: Platanus orientalis and $P$. occidentalis, separated 25 million years ago, still produce fertile hybrids ${ }^{3}$ ).

The relative importance of allopatric and sympatric speciation has yet to be determined. However, the proponents of the latter have already "devised testable predictions to distinguish" it from the former. After allopatric speciation, new species differ in many loci because their ancestors were long isolated. During sympatric speciation, gene exchange continues, and new species retain similar allele frequencies at all loci, except the few responsible for reproductive isolation and ecological divergence ${ }^{2}$. Striking genetic similarity between some sympatric species (for example, cichlids in Lake Victoria $^{4}$ and trouts in Lake Sevan ${ }^{5}$ ) strongly suggests their recent sympatric origin (besides, in these cases there was no room for allopatric speciation).

Dogmatic adherence to the BSC causes much confusion. Of course, it is wrong to carve two species from a panmictic population or to pool into one species sexual forms which, if crossed, never have normal progeny. However, very distinct forms may produce fertile hybrids (including lions and tigers in captivity, and many species of Cyprinidae, Rosaceae and other taxa in nature). Perfectly fertile hybrids can flourish between populations, so different ecologically and morphologically that nobody would consider them the same species ${ }^{6}$, such as Anemone fasciculata and $A$. speciosa in the northern Caucases ${ }^{7}$. Thus, speciation may preceed the genetic isolation mechanisms and for a while depend solely on spatial separation caused by historical factors or different ecology (page 199 of ref. 3).

True, there are 'good' species, including ours, essentially homogeneous and drastically different from relatives, but life is not always that simple. To argue about whether similar but separated populations are already different species is as futile as to discuss how many grains constitute a heap. To draw a sharp border in a gradual cline between two species is also impossible. Even sympatric forms are frequently isolated incompletely (for example species of Papilio ${ }^{8}$ and 'host races' of Lochmaea').

In such cases we cannot discover species like new islands but, instead, face continuous diversity and have to describe it. Still, species, as a more or less distinct and genetically and evolutionary independent entity, is a useful concept, even when applied to asexuals, where ecological factors tend to create discrete forms (page 135 of ref. 10). For sexual organisms the degree of genetic exchange must be important for taxonomic decisions, although the Earth is not populated exclusively by 'good biological species'. This only makes genetics of speciation more exciting.

ALEXEY S. KONDRASHOV

Laboratory of Genetics,

University of Wisconsin, Madison,

Wisconsin 53706, USA

Permanent address: Research Computer Centre, Pushchino, Moscow Region 142292, Russia.

1. Coyne, J. A. Nature 355, 511-515 (1992).

2. Kondrashov, A. S. \& Mina, M. V. Biol. J. Linnean. Soc. 27, 201-223 (1986).

3. Stebbins, G. L. Variation and Evolution in Plants (Columbia University Press, New York, 1950).

4. Meyer, A., Kocher, T. D., Basasibwaki, P. \& Wilson, A. C. Nature 347, 550-553 (1990)

5. Osinov, A. G. Genetika 25, 1827-1835 (1989).

6. Barton, N. H. \& Hewitt, G. M. Nature 341, 497 (1989)

7. Kondrashov, A. S., Onipchenko, V. G. \& Yazvenko, S. B. Bull. Moscow Soc. Naturalists 91, 88-95 (1986).

8. Sperling, F. A. H. Can. J. Zool. 68, 1790-1799 (1990)

9. Kreslavskii, A. et al. Zool. J. 66, 1045-1054 (1987)

10. Fisher, R. A. The Genetical Theory of Natural Selection 2nd edn (Dover, London, 1958).

SIR - Coyne ${ }^{1}$ suggests that the new molecular mapping techniques should provide the data needed to construct models showing how genetic changes lead to speciation. We believe that such studies and models may be more fruitful if it is recognized that inherited epigenetic, as well as genetic, changes may underlie reproductive isolation, particularly in the early stages of speciation.

The importance of inherited epigenetic information is clear from studies of genomic imprinting ${ }^{2,3}$. In many species, genetically identical genes or chromosomes function differently, depending on whether they were inherited from the male or female parent. Chromosomes from mother and father carry different, but complementary, imprints - different epigenetic information. One consequence is that mice that inherit both copies of a chromosome or chromosome region from the same parent are often abnormal. Similarly, mouse embryos constructed from two male or two female pronuclei do not complete development ${ }^{3}$. Although the genetic information they carry is complete and adequate, the epigenetic information is not. To be viable, embryos must have the different, but complementary, epigenetic information from each parent. Incompatibility between the epigenetic information inherited from the parent species could be the cause of the inviability of some species hybrids ${ }^{4}$.

Exactly how chromosomes carry epigenetic information in chromatin structure is not known. In some higher organisms, patterns of DNA methylation are involved,- but DNA-protein interactions are also important ${ }^{2,5}$. Holliday ${ }^{6}$ has pointed out that errors in the transmission of such information (epimutations) are inevitable. Induced epigenetic changes are also possible ${ }^{4}$. The methylation patterns and functioning of particular genes in the mouse are known to differ from strain to strain $^{3}$, and the accumulation of such differences could lead ultimately to inviability or sterility in hybrids between previously isolated populations. Studies of methylation patterns and gene activity in interspecific crosses would help to determine whether this is so. Unfortunately, Drosophila, the genus for which there is most genetic information about speciation, shows little or no DNA methylation.

Recognizing that information carried in chromatin may be important helps to explain some of the observations discussed by Coyne ${ }^{1}$. Faulty chromatin structure has been implicated in some meiotic drive systems, and speciation resulting from cytoplasmic symbionts is associated with defective chromatin condensation. The disproportionately large effect of the sex chromosomes on post-zygotic isolation may be a consequence of the greater changes in chromatin structure that these chromosomes undergo during development, and Haldane's rule may be the result of the more marked chromatin restructuring that takes place in the heterogametic $\operatorname{sex}^{7}$. Because changes in chromatin structure seem to be involved in so many aspects of reproductive isolation, studying the heritable epigenetic information in chromosomes may be necessary if speciation is to be understood.

\section{Cohn Institute for the History and}

Philosophy of Science and Ideas,

Tel-Aviv University,

Tel-Aviv 69978, Israel

MARION J. LAMB

Biology Department

Birkbeck College, Malet Street,

London WC1E $7 \mathrm{HX}$, UK

1. Coyne, J. A. Nature 355, 511-515 (1992).

2. Hoffman, M. Science 252, 1250-1251 (1991)

3. Howlett, S. K. Int. Rev. Cytol. 127, 175-192 (1991)

4. Jablonka, E. \& Lamb, M. J. J. theor. Biol. 139, 69 (1989)

5. Gaunt, S. J. \& Singh, P. B. Trends Genet. 6, 208 (1990)

6. Holliday, R. Science 238, 163-170 (1987)

Jablonka, E. \& Lamb, M. J. Proc. R. Soc. B243, 203-208 (1991) 